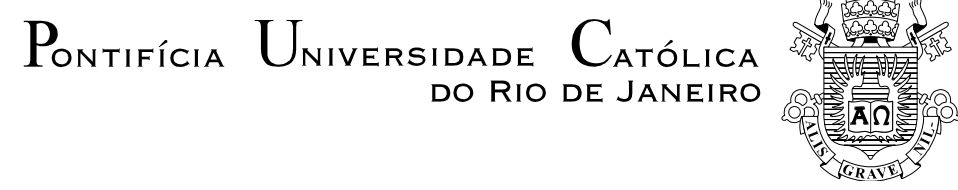

Volney Gilberto Figueiredo Júnior

\title{
Fluxos de Informação em Cadeia de Suprimentos de Derivados de Petróleo: caso Petrobras
}

\section{Dissertação de Mestrado (Opção profissional)}

Dissertação apresentada como requisito parcial para obtenção do título de Mestre pelo Programa de PósGraduação em Engenharia de Produção da PUCRio.

Orientador: Prof. José Eugênio Leal Co-Orientador: Prof. José Roberto de Souza Blaschek 


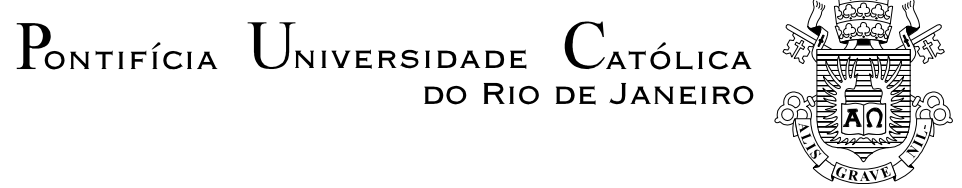

Volney Gilberto Figueiredo Júnior

Fluxos de Informação em Cadeia de Suprimentos de Derivados de Petróleo: caso Petrobras

Dissertação apresentada como requisito parcial para obtenção do título de Mestre pelo Programa de PósGraduação em Engenharia de Produção da PUC-Rio. Aprovada pela Comissão Examinadora abaixo assinada.

Prof. José Eugênio Leal
Orientador
Departamento de Engenharia Industrial - PUC-Rio

Prof. José Roberto de Souza Blaschek Co-Orientador Coordenação Central de Extensão - PUC-Rio

Prof. Gustavo Robichez de Carvalho

Departamento de Informática -PUC-Rio

Prof. Leonardo Guerreiro Azevedo UNI-Rio

Prof. José Eugênio Leal Coordenador(a) Setorial do Centro

Técnico Científico - PUC-Rio

Rio de Janeiro, 15 de agosto de 2012 
Todos os direitos reservados. É proibida a reprodução total ou parcial do trabalho sem autorização da universidade, do autor e do orientador.

Volney Gilberto Figueiredo Júnior

Graduou-se em Engenharia Elétrica pela Pontifícia Universidade Católica do Rio de Janeiro em 1984. Cursou Análise e Projeto de Sistemas na CCE/PUC-RJ em 1985, pós-graduação em Engenharia Econômica na Universidade Estadual do Rio de Janeiro (UERJ) em 1986 e MBA em Logística na Coppead - Universidade Federal do Rio de Janeiro (UFRJ) em 2008. Desenvolveu diversos projetos de tecnologia e sistemas de informação para a área de Logística nas empresas Shell Brasil, IBM e Petrobras.

Ficha Catalográfica

Figueiredo Júnior, Volney Gilberto

Fluxos de informação em cadeia de suprimentos de derivados de petróleo : caso Petrobras / Volney Gilberto Figueiredo Júnior ; orientador: José Eugênio Leal ; coorientrador: José Roberto de Souza Blaschek. - 2012.

117 f. : il. (color.) ; $30 \mathrm{~cm}$

Dissertação (mestrado)-Pontifícia Universidade Católica do Rio de Janeiro, Departamento de Engenharia Industrial, 2012.

Inclui bibliografia

1. Engenharia Industrial - Teses. 2. Cadeia de suprimentos. 3. Derivados de petróleo. 4. Fluxo de informação. 5. Tecnologias aplicadas à logística. I. Leal, José Eugênio. II. Blaschek, José Roberto de Souza. III. Pontifícia Universidade Católica do Rio de Janeiro. Departamento de Engenharia Industrial. III. Título. 


\section{Agradecimentos}

Primeiramente, agradeço a Deus, sem o qual nada disso seria possível.

Aos meus orientadores, pela parceria, estímulo e dedicação durante a realização deste trabalho.

À minha família, que sempre me apoiou e compreendeu a minha ausência em momentos importantes.

Ao corpo gerencial da Petrobras, que entendeu a importância deste curso para o meu crescimento profissional.

Aos professores do curso de mestrado, que me ajudaram a compreender melhor os desafios da logística e gestão da cadeia de suprimentos.

Aos professores que participaram da Comissão examinadora.

Aos meus colegas da Petrobras que sempre me incentivaram e apoiaram. 


\section{Resumo}

Figueiredo Júnior, Volney Gilberto; Leal, José Eugênio; Blaschek, José Roberto de Souza. Fluxos de Informação em Cadeia de Suprimentos de Derivados de Petróleo: caso Petrobras. Rio de Janeiro, 2012. 117p. Dissertação de Mestrado (Opção profissional) - Departamento de Engenharia Industrial, Pontifícia Universidade Católica do Rio de Janeiro.

A integração dos processos e sistemas relacionados à logística dentro das empresas e junto aos seus fornecedores e clientes vem se firmando como uma tendência ao longo dos últimos anos. A utilização da logística como vantagem competitiva e para agregar valor aos produtos e serviços tem se tornado, cada vez mais, um dos direcionadores estratégicos das empresas. Mais recentemente, o planejamento colaborativo passou a ser amplamente utilizado como ferramenta para se obter ganhos de produtividade e custos. No atual ambiente de negócios, o fluxo de informações entre os diversos membros da cadeia de suprimentos, bem como a qualidade e a visibilidade das informações é uma questão fundamental para apoiar a tomada de decisões. O grande desenvolvimento da tecnologia da informação e dos sistemas de informação nas últimas décadas tem contribuído, de forma decisiva, para prover fluxos de informações adequados através dos membros da cadeia de suprimentos. As empresas precisam implantar tecnologias e sistemas de informação que garantam um fluxo de informações ágil e de qualidade a fim de possibilitar uma resposta rápida aos desafios crescentes dos negócios. A dissertação pretende, a partir da fundamentação teórica em cadeias de suprimentos e tecnologia da informação, realizar uma análise da situação atual dos fluxos de informações para o processo de movimentação de derivados da Petrobras, identificar os principais problemas decorrentes do fluxo inadequado de informações, seja em termos de tempo ou em termos de qualidade da informação, e propor soluções de tecnologia e sistemas de informação para tratar os problemas identificados.

\section{Palavras-chave}

Cadeia de Suprimentos; Derivados de Petróleo; Fluxos de Informação; Tecnologias aplicadas à Logística. 


\section{Abstract}

Figueiredo Júnior, Volney Gilberto; Leal, José Eugênio (Advisor); Blaschek, José Roberto de Souza (Co-advisor). Information Flow in Supply Chain for Refined Oil Products: case Petrobras. Rio de Janeiro, 2012. 117p. MSc. Dissertation - Departamento de Engenharia Industrial, Pontifícia Universidade Católica do Rio de Janeiro.

The integration of processes and systems related to logistics inside the companies and together with their suppliers and customers has been established as a trend in the past few years. The use of logistics as a competitive advantage and to add value to the products and services has become, more and more, one of the strategic issues for the companies. More recently, the collaborative planning has been widely applied as a tool to achieve productivity and cost advantages. In today's business environment, the information flow between the supply chain members, as well as the quality and visibility of the information is a key issue to support decision making. The great development of information technology and information systems in the last decades has contributed, decisively, to provide appropriate information flow across the supply chain members. Companies need to set up technologies and information systems that assure an agile and high quality information flow in order to enable a quick reaction to the increasing challenges of business. This work aims to, based on the theoretical foundation of supply chain and information technology, analyse the information flow for the refined oil products process at Petrobras, identify the main issues due to inappropriate information flow, in terms of timing and information quality, and propose technologies and information systems solutions to solve the issues.

\section{Keywords}

Supply Chain; Refined Oil Products; Information Flow; Technologies for Logistics. 


\section{Sumário}

1 Introdução 13

1.1. Justificativa e Motivação 13

1.2. Objetivo Geral 14

1.3. Objetivos Específicos 14

1.4. Metodologia de Trabalho 15

1.5. Organização do Trabalho 15

$\begin{array}{ll}\text { 1.6. Limites do Estudo } & 16\end{array}$

2 Logística, Cadeia de Suprimentos e Fluxos de Informações $\begin{array}{ll}\text { Fundamentação Teórica } & 18\end{array}$

2.1. Logística e Logística Integrada 18

2.2. Cadeia de Suprimentos 22

2.3. Nível de Serviço 25

2.4. Gestão de Estoques 27

2.5. Fluxos de Informações em Cadeias de Suprimentos 28

2.6. Conclusão 34

3 Soluções de Tecnologia aplicadas à Gestão de Informações em Cadeias de Suprimentos $\quad 35$

3.1. Sistemas de Informações Logísticas 35

3.2. Sistemas de Gestão da Cadeia de Suprimentos 38

3.3. Sistemas baseados na WEB $\quad 41$

3.4. Sistemas de Apoio à Decisão (SAD) 43

3.5. Sistemas para o Gerenciamento de Processos de Negócio 45

3.6. Tecnologias e soluções para a coleta e transferência de informações 46

3.7. Tecnologias e soluções para a gestão de dados 48

3.8. Tecnologias e soluções para a apresentação e visualização das informações 50

3.9. Novas Tecnologias 52 
4 Visão Geral da Petrobras e seus Fluxos de Transporte de Produtos 55

4.1. Histórico 55

4.2. A Estrutura Organizacional da Empresa 58

4.3. A Diretoria de Exploração e Produção da Petrobras 59

4.4. A Diretoria de Abastecimento da Petrobras 61

4.5. Principais Fluxos de Transporte de Petróleo 65

4.6. Principais Fluxos de Transporte de Derivados 67

4.7. A Gerência Executiva de Logística 68

4.8. Principais Funções Lógicas do Abastecimento 69

5 Fluxos de Informações e Problemas Identificados 72

5.1. Principais Sistemas que suportam as Funções Lógicas do Abastecimento $\quad 72$

5.2. Elos da Cadeia de Suprimentos da Petrobras 75

5.3. Escopo do Trabalho 77

5.4. Fluxos de Informações da Cadeia de Movimentação de Derivados da Petrobras 80

5.5. Fluxos de Emergências $\quad 87$

5.6. Qualificação dos Problemas 90

5.7. Conclusão 96

6 Apresentação da Solução Proposta 97

6.1. Esboço geral da Solução Proposta 97

6.2. Detalhamento das Soluções Propostas 98

6.3. Valor da Solução Proposta para o Negócio 103

6.4. A Contribuição das Novas Tecnologias 106

6.5. Uma Visão de Arquitetura 107

6.6. Considerações sobre a Implantação das Soluções Propostas 111

7 Conclusões e Recomendações 113

7.1. Recomendações 114 
8 Referências Bibliográficas 


\section{Lista de tabelas}

Tabela 1: Principais problemas resultantes do fluxo inadequado e baixa qualidade de informações em cadeias de suprimento

Tabela 2: Qualificação dos problemas de fluxos de informações

- Parte 1

Tabela 3: Qualificação dos problemas de fluxos de informações

- Parte 2

Tabela 4: Propostas de Soluções para os problemas de fluxos de informações - Parte 1

Tabela 5: Propostas de Soluções para os problemas de fluxos de informações - Parte 2 


\section{Lista de figuras}

Figura 1: Evolução da Logística 18

Figura 2: Fatores que levam à Globalização 19

Figura 3: Modelo Conceitual de Logística Integrada 21

Figura 4: O Modelo de "Supply Chain Management" 23

Figura 5: Relação Genérica entre Vendas e Serviço ao Cliente 26

Figura 6: Reclamações comuns de serviços ao cliente 27

Figura 7: Efeito Chicote $\quad 31$

Figura 8: Aplicações de TI utilizadas em Logística 34

Figura 9: Funcionalidade da Informação 36

Figura 10: Visão Geral do Sistema de Informação Logística 37

Figura 11: Posicionamento dos Sistemas de SCM 39

Figura 12: Fontes de Conteúdo para um Portal de Informações Empresarias $\quad 43$

Figura 13: Fluxo de Informação no Processo de Decisão 44

Figura 14: Ciclo de Vida dos Dados 49

Figura 15: Organização "portalizada" 52

Figura 16: Organograma da Petrobras 58

Figura 17: Macro-processos de Exploração e Produção 59

Figura 18: Cadeia de Valor do Abastecimento da Petrobras 62

Figura 19: Cadeia de Valor do Abastecimento macro-processos de cada Gerência Executiva 63

Figura 20: Refinarias e escritórios Petrobras 64

Figura 21: Refinarias da Petrobras no Brasil 64

Figura 22: Malha de Dutos de Produtos Escuros 66

Figura 23: Malha de Dutos de Produtos Claros 67

Figura 24: Principais blocos de funções lógicas realizadas pelo Abastecimento $\quad 69$

Figura 25: Principais sistemas de informação que suportam os blocos de funções lógicas $\quad 72$

Figura 26: Cadeia de Suprimentos da Petrobras 
(visão simplificada) - modais aquaviário e dutoviário

Figura 27: Escopo do trabalho realizado em relação

aos fluxos de transporte de produtos e sistemas de informação

Figura 28: Fluxos de Programação e Operação na

Movimentação de Derivados

Figura 29: Exemplos de Fluxos de Informações de

Emergências - Parte 1

Figura 30: Exemplos de Fluxos de Informações de

Emergências - Parte 2

Figura 31: Arquitetura de Aplicações em camadas

Figura 32: Arquitetura de Aplicações utilizando somente

Tecnologias Tradicionais

Figura 33: Arquitetura de Aplicações utilizando Novas Tecnologias 